\section{Traumatic perineal injury in a 13-year-old female: Case report and review of the literature}

\author{
Dimitrios Godosis, Christos Kaselas, \\ Charikleia Demiri, Kleanthis \\ Anastasiadis, Theodora Tsiaprazi, \\ Ioannis Spyridakis
}

$2^{\text {nd }}$ Department of Pediatric Surgery. Aristotle University of Thessaloniki, "Papageorgiou" General Hospital, Thessaloniki, Makedonia, Greece

\begin{abstract}
Traumatic perineal injury in children is a rather underestimated condition, in terms of occurrence. The rapid stretching of the soft perineal environment, combined with the surrounding osseous tissue of the pelvis, can cause severe injuries. The key to successful management of these injuries includes timely resuscitation, thorough physical examination and quick and safe repair of damages.
\end{abstract}

\section{Introduction}

Perineal trauma in pediatric patients is not a common condition, with a reported incidence of 4-6\% in injured children of any etiology. 1,2 The rapid and excessive stretching of the soft perineal tissues against the fixed bone structures of the pelvis may cause different types of injuries. We present a case of a girl referred to our department after a blunt perineal injury accident.

\section{Case Report}

\section{History of present illness}

A 13-year old girl was referred to our department from a peripheral hospital after traumatic perineal injury with a rolling pin. According to medical history, the patient landed on the rolling pin while doing some dancing training for a school play. She was taken to the local referral hospital where a perineal laceration was identified. Due to the nature of the wound, absence of symptoms and further clinical findings at initial clinical evaluation, as well as lack of a pediatric surgeon, the child was initially treated by the hospital's gynecological team. She was taken to theatre for wound investigation and, according to their report, it was a penetrating wound that luckily did not injure neither the external genitalia, nor the anal sphincter. In the absence of further findings treatment was limited to suturing of the skin laceration.

The child was followed as an inpatient and remained symptom-free for a few hours; however, a massive bloody bowel movement raised significant concerns of the course of her treatment and a transfer to our department was arranged.

\section{Physical examination}

On arrival, the child was in a good general condition with normal vital signs; laboratory studies revealed a drop of hematocrit to $27.1 \%$ with hemoglobin of $10.2 \mathrm{~g} / \mathrm{dL}$. On physical examination the abdomen was soft without any clinical signs of peritoneal irritation; a rectal examination was performed that revealed fresh blood in the rectum indicative of active bowel bleeding and a feeling of disruption of anterior bowel wall integrity. Due to high suspicion of colorectal injury an abdominal X-ray was performed that did not reveal any indications of bowel rupture or peritoneal contamination. Patient was scheduled and taken to theatre as soon as possible. A colonoscopy preceded any surgical intervention that identified an actively-bleeding anterior bowel wall laceration $2 \mathrm{~cm}$ above the dentate line. No further damage to the rest of the bowel was noted.

At that point, a wound investigation was decided and performed. The skin laceration was just lateral and to the right of the anus. The vagina and the anal sphincter were intact but we found that the wound was extending deep enough in an oblique fashion, leading to a 2-cm-long laceration of the bowel wall; on simultaneous rectal digital examination we were surprised that the surgical glove from the digit performing the rectal examination could be seen through the perineal wound verifying the severity of the bowel injury.

Closure of the bowel injury was performed in two stages; seromuscular closure was performed through the perineal wound while bowel mucosa was closed transanally. Finally, a protective colostomy was conducted following laparotomy for inspection of the peritoneal cavity.

The patient had an uneventful postoperative period and colostomy closure was performed 3 months later. After a six-month follow up period the patient is doing well with normal bowel movements.

\section{Discussion}

Perineal injuries in female children is a
Correspondence: Dimitrios Godosis, 2nd Department of Paediatric Surgery, Aristotle University of Thessaloniki, "Papageorgiou" General Hospital, Thessaloniki Ring Road, Nea Efkarpia Region, 56403, Thessaloniki, Makedonia, Greece.

Tel.:+30.2313323547, +30.6945682340

E-mail: konpal1453@yahoo.gr

Key words: perineal trauma, laceration, children, pediatric surgery.

Contributions: DG: data collecting, analyzing, manuscript writing, final revision; CK: data analyzing, manuscript writing; $C D$ : references search, final revision; KA: references search, final revision; TT: manuscript writing; IS: references search, final revision.

Conflict of interest: the authors declare no potential conflict of interest.

Funding: none.

Received for publication: 19 Januaray 2019

Revision received: 27 May 2019.

Accepted for publication: 28 May 2019.

This work is licensed under a Creative Commons Attribution NonCommercial 4.0 License (CC BY-NC 4.0).

CCopyright D. Godosis et al., 2019

Licensee PAGEPress, Italy

Pediatric Reports 2019; 11:7993

doi:10.4081/pr.2019.7993

rare condition, with a reported incidence of $4-6 \% .^{1}$ The main cause in childhood is impalement. ${ }^{3}$ However in cases of vaginal injury sexual abuse has to be excluded. ${ }^{4}$ All children with these injuries should be suspected as sexual assault victims and be routinely referred to the social worker and/or relevant law enforcement authorities.

The trauma mechanisms can be described as straddle (following falling or sitting on the perineal area with the legs open), non-straddle blunt (trauma from outside factors other than falling - ball or stone), laceration (trauma that causes a cut or laceration in the genital and perineal area due to falling or an outside factor) and coital (trauma caused by sexual intercourse whether penetration occurred or not). ${ }^{5}$

According to Narang et al., perineal tears in children have been given little attention as compared to perineal injuries following child birth in adult females. ${ }^{3}$ Therefore, the classification proposed by Sultan et al for perineal tears of females during childbirth is readily used (Table 1). ${ }^{6}$

Endoscopic evaluation under general anesthesia is the first line approach, when referring to perineal traumas. Proctoscopy 
(followed by rectoscopy when appropriate) is necessary, as an accurate evaluation of the severity of the perineal injury and a possible sphincter or/and rectal co-injury should be evaluated.5,7 The possibility of a serious rectal or sphincteric injury should not be underestimated, as such kinds of impairments in case of communication with the peritoneal cavity can lead to peritonitis and septic shock if left untreated. Proctoscopy is mainly carried out for the assessment of the integrity of the sphincteric mechanism, namely the internal and external sphincter muscles. Moreover, if full thickness disorganization in the proximal two-thirds of the rectum is detected, it should be considered that the internal sphincter is breached. ${ }^{8}$ Visual inspection during proctoscopy should also be accompanied with palpation for the integrity of the sphincteric mechanism to be assessed, because submucosal lesions with an intact mucosa would not be visually confirmed. 9,10

Male and female patients with such injuries should furthermore undergo a cystoscopy, especially in the presence of macroscopic hematuria and/or anterior rectal wall impairment. ${ }^{10}$ In terms of impalement lesions in young girls, an additional vaginoscopy could be performed for vaginal injuries to be excluded. ${ }^{11}$

After endoscopy is completed and a suspicion of intra-abdominal extension of the lesion is confirmed, an exploratory laparotomy is the gold-standard approach, being used as diagnostic (for exclusion of intra-peritoneal involvement) and also therapeutic tool, with or without a subsequent diversion (protective) colostomy. ${ }^{7}$

Surgeons should be prepared to submit swabs, aspirates and washings from all cavities taken at the time of proctoscopy and vaginoscopy for culture and microscopy, ${ }^{12}$ semen analysis and DNA analysis if necessary. Detailed operative notes ${ }^{12}$ and diagrams should be drawn in the event of court action. Especially wounds caused after impalement are difficult to primarily estimate, due to the usual small area of the external lesion, in comparison with the extent of the internal injury. ${ }^{13}$

Supplementary, but also elementary diagnostic tools in the management of perineal injuries include radiologic investigation and CT scan. An X-ray of the abdomen (especially combined with an erect chest Xray) should give us the image of intraperitoneal free air, an indication that the injury has extended in the peritoneal cavity. Barium as a contrast enema must not be used as there is a certain probability for barium peritonitis with concomitant electrolyte balance abnormalities. ${ }^{14} \mathrm{CT}$ scan has an indication of usage in terms of identifying rectal injuries missed on initial proctoscopy, due to poor visualization or an extremely unprepared rectum, accompanied with a triple contrast solution (oral, rectal and intravenous). 15

Once initial management (as mentioned above) is performed, the classic approach in terms of fecal diversion is a protective loop sigmoid colostomy. Fecal diversion is applicable in intraperitoneal injuries, as well as in extraperitoneal ones, when there is a large scale of perineal trauma and a high risk of secondary infection to the impaired tissues. ${ }^{12}$ The type of colostomy widely approved is loop colostomy, in opposition of divided colostomies. The reason of this traditional way of management is multiple: easy construction, easier closure, adequate fecal diversion (allowing adequate healing) for the purpose of perineal and low rectal injuries. ${ }^{16}$

As an adequate healing of the bowel requires about 7-11 days following trauma, ${ }^{17}$ there is a tendency of "same admission colostomy closure". ${ }^{13}$ However, a traditional approach is nowadays of common practice, which indicates closure of colostomy in about 6 weeks after injury, while no adverse effects have been noticed. ${ }^{18}$ A prior to closure loop-ogram is controversial in terms of its actual usefulness, as there is not always a high yield for this examination. However, it proves useful in indicating severe strictures in the aferrent part of the bowel prior to closure of the colostomy.

In the case of impalement, location of entry wound as well as angle and intensity of penetration affects the trajectory of the object and impacts adjacent organs involvement. Inspecting the penetrating object is advisable as its characteristics may influence the work-up approach.

In terms of anorectal injuries, management is quite similar to that of adults. It includes fecal diversion (colostomy), presacral drainage and antibiotic chemoprophylaxis.

Regarding our case, some clarifications are needed. According to history of trauma, there was indeed a suspicion of anorectal injury. Thorough examination (colonoscopy, vaginoscopy) revealed a 2$\mathrm{cm}$ long laceration of the bowel wall, as it was above mentioned, located about $2 \mathrm{~cm}$ above the dentate line and a concomitant communication between the anterior colonic wall and posterior vaginal wall. The exclusion of intraabdominal involvement (at first with an erect abdominal X-Ray and secondly through exploratory laparotomy) in this case was not a prohibitive factor for a fecal diversion to be performed. In this case we preferred to follow the diversioncolostomy-approach, despite the extraperitoneal nature of the laceration, because there was a high possibility of urogenital contamination, due to the direction of the impairment from the vagina towards the anal canal.

Although there are algorithms suggesting fecal diversion only in cases of intraperitoneal involvement, ${ }^{14}$ a loop colostomy is not excluded when high possibility of contamination of adjacent tissues does exist. ${ }^{13}$ Moreover there is a controversy in terms of necessity of fecal diversion in extraperitoneal rectal injuries. ${ }^{19}$ Generally, fecal diversion is proposed in cases of lifethreatening trauma, substantial associated injuries, large scale perineal involvement and high probability of generalized contamination. ${ }^{20}$ In our case, the diversion-colostomy-approach was preferred in order to rule out possible future complications from urogenital system, while simultaneously providing a safe, unpolluted environment for the injured anal section to be healed.

Table 1. Sultan's classification of perineal tears in females during childbirth.

\begin{tabular}{ll} 
Degrees & Definition \\
1 & Laceration is limited to the fourchette and superficial perineal skin or vaginal mucosa \\
2 & Laceration extends beyond fourchette, perineal skin and vaginal mucosa to perineal muscles and fascia, but not the anal sphincter \\
\hline $3 \mathrm{a}$ & Partial tear of the external anal sphincter involving less than $50 \%$ thickness \\
\hline $\mathrm{b}$ & Greater than $50 \%$ tear of the external anal sphincter \\
\hline $3 \mathrm{c}$ & Internal sphincter is torn \\
4 & Fourchette, perineal skin, vaginal mucosa, muscles, anal sphincter, and rectal mucosa are torn \\
\hline
\end{tabular}




\section{Conclusions}

Perineal trauma in pediatric patients is a rather rare condition, with a reported incidence of $4-6 \%$ in injured children of any etiology. The main cause is impailment. It is always mandatory that sexual abuse scenarios have to be excluded. It is of optimum importance to organize the type of management, depending on the mechanism, the location of entry and the involvement of adjacent organs. As occult injuries may not be visible during primary clinical assessment, endoscopic evaluation, including rectoscopy cystoscopy and vaginoscopy in female patients, should be performed in all cases of major perineal traumas. Fecal diversion should not be avoided in cases necessary for the patient's survival and long-term uncomplicated outcome, while extra measures should be taken in terms of chemoprophylaxis, due to risk of contamination.

\section{References}

1. Bond GR, Dowd MD, Landsman I, Rimsza M. Unintentional perineal injury in prepubertal girls: a multicenter prospective report of 56 girls. Pediatrics 1995;95:628-31.

2. Spitzer RF, Kives S, Caccia N, et al. Retrospective review of unintentional female genital trauma at a pediatric referral centre. Pediatr Emerg Care 2008;24:831-5.
3. Narang V, Sen A, Aggarwal S. Traumatic perineal injuries in prepubertal girls: a review of five cases. Indian J Basic Appl Med Res 2016;6:293-9.

4. Beiler HA, Zachariou Z, Daum R. Impalement and anorectal injuries in childhood: a retrospective study of 12 cases. J Pediatr Surg 1998;33:1287-91.

5. Kaplanoglu M, Demirkıran DS, Karateke A, Arslan MM. Mechanism and treatment of genital injuries in pediatric and adolescent girls: data from two regional centers in Turkey. J Forensic Investigation 2014;2: 3.

6. Sultan AH, Thakar R. Lower genital tract and anal sphincter trauma. Best Pract Res Clin Obstet Gynaecol 2002;16:99-115.

7. Bonnard A, Zamakhshary M, Wales PW. Outcomes and management of rectal injuries in children. Pediatr Surg Int 2007;23:1071-6.

8. Delancey JO, Toglia MR, Perucchini D. Internal and external sphincter anatomy as it relates to midline obstetric lacerations. Obstet Gynecol 1997;90:924-7.

9. Reinberg O, Yazbeck S. Major perineal trauma in children. J Pediatr Surg 1989; 24:982-4.

10. Jona JZ. Accidental anorectal impalement in children. Pediatr Emerg Care 1997;13:40-3.

11. Grisoni ER, Hahn E, Marsh, et al. Pediatric perineal impalement injuries. J Pediatr Surg 2000;35:702-4.

12. Jones LW, Bass DH. Perineal injuries in children. Br J Surg 1991;78:1105-7.
13. Vincent MV, Abel C, Duncan ND. Penetrating anorectal injuries in Jamaican children. Pediatr Surg Int 2012:1101-7.

14. Hernanz-Schulman M, Foster C, Maxa $\mathrm{R}$ et al. Experimental study of mortality and morbidity of contrast media and standardized fecal dose in the peritoneal cavity. Pediatr Radiol 2000;30:369-78.

15. Leaphart CL, Danko M, Cassidy L, et al. An analysis of proctoscopy vs computed tomography scanning in the diagnosis of rectal injuries in children: which is better? J Pediatr Surg 2006;41:700-3.

16. Tuggle D, Huber PJ Jr. Management of rectal trauma. Am J Surg 1984;148:8068.

17. Blomquist P, Jiborn H, Zederfeldt B. Effect of diverting colostomy on collagen metabolism in the colonic wall. Am J Surg 1985;149:330-3.

18. Bronkhorst MW, Wilde JC, Hamming JF, Heij HA. Anorectal impalement in a pediatric patient with transanal evisceration of small bowel. J Pediatr Surg 2007;42:E23-5.

19. Gonzalez RP, Phelan H 3rd, Hassan M et al. Is fecal diversion necessary for nondestructive penetrating extraperitoneal rectal injuries? J Trauma 2006;61:815-9.

20. Samuk I, Steiner Z, Feigin E, et al. Anorectal injuries in children: a 20-year experience in two centers. Pediatr Surg Int 2015;31:815-9. 